Résumés des conférences et travaux

\title{
Numismatique et économie monétaire de l'Occident médiéval et moderne
}

\author{
Marc Bompaire
}

\section{CpenEdition}

Journals

Édition électronique

URL : https://journals.openedition.org/ashp/2444

DOI : $10.4000 /$ ashp.2444

ISSN : 1969-6310

Éditeur

Publications de l'École Pratique des Hautes Études

\section{Édition imprimée}

Date de publication : 1 septembre 2018

Pagination : 201-212

ISSN : 0766-0677

\section{Référence électronique}

Marc Bompaire, « Numismatique et économie monétaire de l'Occident médiéval et moderne »,

Annuaire de l'École pratique des hautes études (EPHE), Section des sciences historiques et philologiques

[En ligne], 149 | 2018, mis en ligne le 11 juillet 2018, consulté le 03 août 2021. URL : http://

journals.openedition.org/ashp/2444 ; DOI : https://doi.org/10.4000/ashp.2444 


\title{
NUMISMATIQUE ET ÉCONOMIE MONÉTAIRE DE L'OCCIDENT MÉDIÉVAL ET MODERNE
}

\author{
Directeur d'études : M. Marc BompaIRE
}

Programme de l'année 2016-2017 : I. Le monnayage au Moyen Âge, les techniques et les mots. - II. Ouvrages et travaux récents en numismatique médiévale.

I. Après une séance d'introduction les conférences ont été largement liées aux activités et enquêtes du DE. Elles ont d'abord porté sur les mines d'argent avec une lecture de codes, règlements et contrats miniers à la suite du colloque Les métaux précieux en Méditerranée au Moyen Âge, Aix, octobre 2016 auquel il a participé. La publication de l'ouvrage collectif Il tesoro di un povero. Il memoriale di Francesco Bentaccordi, fiorentino in Provenza, dir. J. Hayez, S. Brambilla, Rome, 2016, auquel a contribué le DE a été l'occasion d'une présentation et une réflexion sur les « praticas » ou livres de marchands italiens puisque ce manuscrit contient un texte inédit et probablement rédigé pour une part à Montpellier vers 1361, ce qui en fait un document particulièrement précieux pour l'économie et la circulation monétaire en Languedoc et en Catalogne, un aspect présenté par le DE lors d'une communication à Perpignan en juin 2017. Une séance a été plus spécifiquement consacrée à l'exploitation des trésors monétaires sur lesquels le DE a présenté une communication en avril à Erbil, mais aussi à l'occasion de la publication consacrée par François de Callataÿ aux rythmes des thésaurisations. De même, à l'occasion d'une intervention du DE sur la crise de grande inflation des années 1419-1421 lors d'un colloque à Prague en mai, quelques séances ont permis d'approfondir la typologie des crises monétaires à partir d'une relecture de l'ouvrage dirigé par Bruno Théret, La monnaie dévoilée par ses crises, Paris, 2007. La question des faux monétaires a également été abordée en écho à une journée d'étude co-organisée par le DE en avril sur « analyses métalliques et faux monétaires ». M. Lagasse, auditeur, a présenté à ce propos un dossier sur un supplice peu connu pour des femmes convaincues de faux monnayage qui furent enterrées vivantes à Abbeville au $\mathrm{XV}^{\mathrm{e}}$ siècle.

Quelques points ont retenu plus longuement l'attention des participants au séminaire : les liaisons de coins et leur mode de représentation ont fait l'objet d'interventions de G. Sarah, chercheur CNRS, S. Achache, doctorant et B. Foucray, conservateur général de l'archéologie, à l'occasion de présentations d'études de trésors du haut Moyen Âge. Cette enquête se prolonge dans un projet de recherche "Visualcoins » financé par la MSH Centre Val-de-Loire et porté par Guillaume Sarah. Des interventions de G. Peigney, V. Borrel, doctorants ont présenté l'avance de recherches sur des monnayages féodaux du Centre et du Sud-Est.

La lecture monétaire de la récente publication de Michel Jones et Philippe Charon ${ }^{1}$ a ouvert la réflexion sur deux questions. La question des évaluations monétaires au

1. M. Jones, P. Charon, Comptes du duché de Bretagne. Les comptes, inventaires et exécution des testaments ducaux 1265-1352, Rennes, 2017. 
temps de Philippe le Bel sera reprise et approfondie dans les séminaires ultérieurs car ces comptes laissent apparaître des évolutions plus complexes qu'il ne ressortait de leurs éditions anciennes et partielles. Les inventaires des trésors ducaux illustrent les vastes thésaurisations princières sur lesquelles il apparaît souhaitable de mener une étude comparative. Le DE avait présenté l'extraordinaire masse de numéraire décrite dans l'inventaire après décès de Sully en 1642 qui vient d'être édité par Bernard Barbiche ${ }^{2}$ mais un élargissement de l'enquête vers l'examen d'autres inventaires médiévaux de trésors pontificaux ou royaux, comme les coffres de Charles $\mathrm{V}$, devrait permettre de mieux comprendre les rythmes de constitution et d'utilisation de tels ensemble sur des durées variables et parfois réduite ainsi que leurs effets possibles sur la circulation monétaire.

\section{Les monnaies féodales bléso-chartraines}

L'ouvrage récent de Sylvain Michon, Les monnaies féodales bléso-chartraines, Wetteren, 2016 (collection Moneta) a été l'occasion de présenter cet ensemble de monnayages et d'examiner plus particulièrement la dernière période de leur existence, le $\mathrm{XIII}^{\mathrm{e}}$ et le début $\mathrm{du} \mathrm{XIV}^{\mathrm{e}}$ siècle. Les débuts de ces émissions au $\mathrm{X}^{\mathrm{e}}$ siècle avaient été abordés au cours des années antérieures lors de l'étude de trésors comme le trésor dit « du Loiret» en cours de publication ${ }^{3}$.

Il y a lieu, en effet, de relever le caractère original de ces monnayages reconnaissables au premier coup d'œil, sans que l'on puisse véritablement déterminer s'ils forment un ensemble monétaire, économique ou politique, ce que rien dans les textes de l'époque ne vient étayer. Ils forment un ensemble numismatique fondé sur une typologie commune. Sans évoquer leurs interprétations anciennes qui jouèrent avec l'orientation de la tête quadrangulaire pour la transformer en substitut du «chatel tournois ", le type des monnaies royales du système tournois, ou pour l'inscrire sur les armes de la ville de Chartres ou broder sur la tradition qui y a cherché une représentation de la relique chartraine de la chemise de la Vierge, on relève en effet les difficultés des auteurs à décrire ce type sans trop l'interpréter: monnaies au type chartrain selon E. Cartier en $1846^{4}$, au type chinonais selon G. de Ponton d'Amécourt en $1895^{5}$ qui s'appuie sur la position de F. Poey d'Avant ${ }^{6}$, alors qu'E. Caron précisait qu'il serait plus naturel de désigner ce type sous le nom de tête Turono-chinonaise ${ }^{7}$.

2. B. Barbiche, Le Château de Villebon en 1642. Inventaire après le décès de Maximilien de Béthune, duc de Sully, Paris, CTHS, 2017.

3. S. Achache, M. Bompaire, A. Castelas, « Le trésor dit "du Loiret”, présentation, catalogue et étude caractéroscopique », Trésors monétaires XXVII. Monnayages de Francie des derniers Carolingiens aux premiers Capétiens, Paris, 2018, p. 1-99.

4. E. Cartier, " Essai sur les monnaies au type chartrain », Revue numismatique, 1844, p. 405-428, 1845, p. 26-51, 112-141, 196-226, 275-308, 360-395 et 1846 p. 28-55, 107-133.

5. G. de Ponton d'Amécourt, "Description générale des monnaies au type chinonais », Annuaire de la Société française de numismatique, 1895 p. 137-163, 242-260, 359-381, 426-462, 540-571.

6. F. Poey d'Avant, Monnaies féodales de France, t. 1, 1858.

7. E. Caron, Monnaies féodales françaises, Paris, 1882. 
Le terme de «monnaies bléso-chartraines », popularisé par le Manuel de A. Dieudonné $^{8}$ est repris par S. Michon. Celui-ci en fait un emblème thébaldien de la famille des comtes de Blois et Chartres (et de Champagne) : « Il faut donc voir dans cette tête une marque personnelle de la famille, sorte d'armoirie avant la lettre ». Une terminologie plus neutre : type anépigraphe à la tête a été proposée par M. Dhénin ${ }^{9}$ plus ou moins suivi par P. Crinon ${ }^{10}$ ou R. A. Merson ${ }^{11}$.

Les monnaies bléso-chartraines ont particulièrement recueilli l'attention des premières générations de spécialistes des monnaies françaises, à commencer par les deux créateurs de la Revue de la Numismatique Françoise, en 1836, Louis de La Saussaye, de Blois et Étienne Cartier, de Tours, qui leur avait consacré une étude que S. Michon décrit comme la « doctrine » numismatique et qu'il « relègue au rang d'hérésie »... « que l'ethnographie structuraliste condamne ». C'est une façon de présenter l'historiographie du sujet. À la génération suivante, Gustave de Ponton d'Amécourt, esprit curieux et créateur du terme hélicoptère, appartient à la même catégorie des aristocrates numismates que S. Michon décrit comme assez naïfs pour prendre pour argent comptant des trésors fabriqués à leur intention. Il écarte ainsi de la liste des trésors fiables les trouvailles de la «butte de Chartres » (y compris le petit groupe de celles qui ont été signalées comme formant un ensemble) ou le trésor assurément complexe sinon composite de Beaugency et généralise : "Il en va de même, à notre sens, de l'ensemble des trésors publiés par Cartier », en y ajoutant les trésors de Bourré et de Saint-Aignan...

Sur cette table rase S. Michon apporte le recensement du matériel qu'il a inventorié, au Musée de Chartres tout d'abord puis également dans d'autres collections, ce qui permet de repérer les types rarissimes parmi ceux qui forment l'essentiel du monnayage.

Son travail se fonde sur des intuitions dont la démonstration reste hors de portée : une « analogie que j'avais cru remarquer entre les monnaies de Troyes et les monnaies de Champagne » [et celles de Blois et Chartres]... le conduit à une hypothèse affirmée nettement et considérée comme une donnée : « la concordance observée dans le temps entre les types monétaires de deux régions distinctes, originairement possession de la même famille... ne pouvait signifier qu'une union économique symbolisée par une union monétaire ». Cette hypothèse, couplée avec un fait, «l'alignement en 1224 du denier provinois de Champagne sur le denier tournois royal », « éclairait d'un jour nouveau quelques vieux problèmes historiques comme la fonction financière des foires de Champagne ou la crise monétaire du règne de Philippe IV le Bel ».

8. A. Dieudonné, Manuel de numismatique française, t. IV. Les monnaies féodales, Paris, 1936, reprenant la question depuis J. Lellewel, Numismatique du Moyen Âge considérée sous le rapport du type, Paris, 1835 .

9. M. Dhénin, «Un denier de Chartres au type anépigraphe à la tête », Club français de la médaille, 58 ( $1^{\text {er }}$ trimestre 1978), p. 154-158.

10. P. Crinon, « À propos de deniers inédits de Blois ( $\mathrm{X}^{\mathrm{e}}$ siècle) : le monnayage à la tête dans les domaines de Thibaud de Tours et Thibaud $\mathrm{I}^{\mathrm{er}}$ (durant les deux premiers tiers du $\mathrm{x}^{\mathrm{e}} \mathrm{s}$.) », BSFN 48, 1 (janvier 1993), p. 467-472.

11. R. A. Merson, «Une monnaie rare retrouvée : le denier à la tête dite "chinonaise" ou anépigraphe (PA 212) à attribuer à Nantes $\mathrm{X}^{\mathrm{e}}$ siècle », BSFN, 52, 6 (juin 1997), p. 122-123. 
Malheureusement les quelques pistes proposées pour tester cette hypothèse sont à peine esquissées. Il n'y a aucun indice que les termes de «provinois » puis de « tournois » aient jamais dans les sources écrites désigné les monnaies « bléso-chartraines ». Inversement les trouvailles de provinois en France (surtout dans l'Est) comme en Italie ne sont pas insignifiantes ni assurément inférieures en nombre à celles des monnaies « bléso-chartraines ».

L'observation dans les deux monnayages de Champagne et Chartres d'un passage des besants à une croisette comme meubles du type principal est interprétée comme marquant une nouvelle émission lancée simultanément. D'autres meubles comme le lis ou la rosette (ou l'étoile) qui apparaissent ensuite sur plusieurs monnayages bléso-chartrains sont interprétés de même comme des jalons pour la datation d'autant d'émissions simultanées, mais aussi comme le signe de l'existence d'un système monétaire organisé et cohérent où les pièces des divers ateliers correspondent à une même valeur, au-delà de leur apparente multiplicité. L'hypothèse mérite assurément l'attention, d'autant que les comtés de Blois et Chartres se retrouvent alors réunis par Jean puis Jeanne de Chatillon mais elle est développée de façon très systématique et déclinée de façon subtile, si subtile qu'il devient difficile de discerner au premier coup d'œil ce qui rapproche les pièces des divers ateliers rattachées à une même émission, même à l'époque de Philippe le Bel où les contenus métalliques ont pu fortement varier d'une émission à l'autre -pour autant que les différentes autorités émettrices se soient efforcées d'aligner leurs émissions sur celles du roi. Pour argumenter à l'appui de cette hypothèse, des analyses métalliques seraient particulièrement utiles. En effet, l'examen des mentions monétaires et des équivalences entre monnaies des divers ateliers n'apporte que peu d'éléments. C'est ensuite l'étude des trésors monétaires qu'il convient de reprendre. Ceux-ci ne sont pas recensés par S. Michon sinon pour appuyer en annexe une autre intuition, une autre hypothèse, sur le rôle particulier joué à Chartres au XII ${ }^{\mathrm{e}}$ siècle par les nombreuses frappes d'oboles qui auraient été destinées à la rémunération des tâcherons du chantier de la cathédrale. Cette idée est d'autant plus séduisante qu'elle rejoint les pistes explorées à partir d'autres exemples par Thibault Cardon dans sa thèse soutenue en $2016^{12}$. Mais là encore l'ouvrage ne fournit pas les données permettant véritablement de vérifier ou d'étayer l'hypothèse.

Pour discuter les propositions de classement de chronologie avancées par S. Michon, il est peu de textes sur lesquels s'appuyer, en dehors de quelques comptes pour des émissions tardives de Blois ${ }^{13}$, mais la liste des titres de divers monnayages copiée par F. Bentaccordi sur un document datable des années 1310 vient ajouter des données nouvelles sur une période proche du célèbre règlement de 1315 sur le monnayage des barons. Plusieurs émissions avec des conditions d'émission variables et une surreprésentation des oboles se succèdent sur une brève période à Blois et des négligences et des fraudes élargissent encore la gamme des valeurs sans que l'on puisse savoir dans lesquels de ces cas des marques étaient apposées sur les pièces pour permettre leur différenciation. La date d'arrêt des fabrications est en revanche

12. T. Cardon, Les usages des monnaies (mi XII -début XVI ${ }^{e}$ s.). Pour une approche archéologique, anthropologique et historique des monnaies médiévales, thèse, 2016.

13. M. Bompaire, « La Monnaie comtale de Blois au début du XIV siècle », BSFN, 39, mars 1984, p. 460464. 
bien établie puisque la plupart de ces monnayages sont vendus au roi de France dans les années entre 1318 et 1328.

La réflexion s'est davantage fondée sur l'examen des trésors monétaires en intégrant un certain nombre de trésors qui n'avaient pas été recensés par Jean Duplessy ${ }^{14}$. Il s'agit en particulier de trésors trouvés hors de France comme les trésors mêlant gros tournois d'argent et deniers de billon trouvés à s-Hertogenbosch (Bois-Le-Duc aux Pays-Bas), enfoui vers 1305 car il compte un nombre important de doubles (parisis) et publié en 2017 par Jan Pelsdonk ${ }^{15}$ ou le gros trésor trouvé à Bonn en 1962 mais dont l'inventaire a été édité en 2015 seulement par E. Auer ${ }^{16}$ et a fait l'objet d'un commentaire de M. Phillips ${ }^{17}$ pour lequel le terminus semble double : vers 1306 plutôt que 1302 pour le pot contenant les monnaies d'argent et vers 1290 plutôt que 1296 pour les deniers de billon. La présence éventuelle d'une seule pièce avec un 0 long postérieure à 1290 (une obole incertaine) et plus sûrement celle d'une obole d'Angers de Charles de Valois (1290-1325) fixe le terminus de cet ensemble de deniers, à condition que le double parisis (après 1296) très usé qui est décrit dans le catalogue soit issu du pot contenant les monnaies d'argent. Ces deux trésors renfermaient un certain nombre de monnaies bléso-chartraines et témoignent de la large circulation de ces pièces aux côtés des deniers tournois du roi de France.

Des trésors sont également apparus en France depuis le travail de J. Duplessy : certains sont anciens et ont été signalés, dans diverses enquêtes comme le trésor de Bressuire dans celle d'Arnaud Clairand ${ }^{18}$. D'autres trouvailles, récentes, ont été publiées, par exemple le trésor de Saint-Léger-des-Bois (Maine-et-Loire) étudié par Magali Heppe ${ }^{19}$, qui serait à dater d'avant 1295 plutôt qu'entre 1307 et 1310 (du fait de l'absence des émissions affaiblies de doubles). Certains sont restés inédits comme le trésor de Saint-Valery-en-Caux (Seine-Maritime) enfoui vers 1310, rapidement dispersé sur internet dans les années 2000 et signalé en 2006 par Yannick Jézéquel ${ }^{20}$ qui a recueilli l'essentiel de la documentation photographique qu'il a présentée au séminaire.

C'est aussi le cas du trésor de Mansigné (Sarthe) qui a fait l'objet en 2016 d'un rapport détaillé de Tiphaine Bellat pour le compte du service régional de l'archéologie et dont le DE a été appelé à faire une expertise permettant de situer son terminus vers 1303-1306. L'étude de T. Bellat avait identifié avec exactitude les exemplaires,

14. J. Duplessy, Les trésors monétaires médiévaux et modernes découverts en France, 2 vol., Paris, 1985, 1995.

15. J. Pelsdonk, "The s-Hertogenbosch1998 Hoard: treasure behind the gilded armour », dans Money and its Use in medieval Europe Three Decades On, Essays in honour of Professor Spufford, M. Allen N. Mayhew (éd.), Londres, 2017, p. 43-49.

16. Die Turnosgroschen aus dem Münzschatz von Boeselagerhof Bonn, Eberhard Auer (éd.), Bonn, 2015, (Bonner Numismatik Studien 2), 375 p.

17. M. Phillips, «The Bonn Hoard. Review article », Numismatic Chronicle, 177 (2017), p. 536-572.

18. Argent caché... et retrouvé. Les trésors monétaires découverts en Deux-Sèvres, M. Cavaillès, A. Clairand, C. Gendron, J. Hiernard, F. Téreygeol, Niort, 2007.

19. M. Heppe, «Étude du trésor médiéval de Saint-Léger-des-Bois (49)», Armornumis, 123 (2008), p. 1-18.

20. Y. Jézéquel, « Monnaies ducales de Bretagne dans un trésor normand (1307-1311)», BSFN, 61, 6 (juin 2006), p. 139-148. 
une tâche délicate dans la mesure où le trésor n'a pas bénéficié d'un nettoyage ni d'une restauration. En conséquence, la couverture photographique est très partielle avec un peu moins d'un exemplaire de chacun des types et variétés recensés. Cela risque de constituer un frein à l'exploitation future de ce trésor dans la mesure où la date proposée pour l'abandon de ce trésor doit être révisée, ce qui n'est pas sans donner davantage de relief à certaines des émissions figurant dans le trésor. En effet les monnaies de Bourgogne au nom de Hugues ne sont pas attribuables à Hugues V (1305-1315) comme le montre l'ouvrage de référence de Françoise Dumas ${ }^{21}$, mais à Hugues IV et les pièces de ce type 8-1-2 circulent dans les années 1255 et surtout autour de 1285. De même, le classement des émissions des ducs Jean de Bretagne a été révisé en 2006 par Y. Jézéquel à la lumière du trésor de Saint-Valery et la monnaie du trésor de Mansigné (type Duplessy 99, Jézéquel 83), d'ailleurs fort usée, attribuée jusqu'alors à Jean III (1312-1320) est ainsi à replacer à la fin du règne de Jean II dans les années 1298-1303.

Des monnaies nouvelles sont également apparues qui viennent enrichir le corpus présenté par S. Michon. Celui-ci, grâce à la photographie des pièces elles-mêmes, offre le grand avantage de permettre de repérer des cas de reprise de types anciens qui n'apparaissaient pas sur des gravures au trait.

\section{Chartres}

Pour Chartres, S. Michon distingue ainsi un type nouveau de Charles de Valois avec deux petits annelets accostant la « chevelure » sur une monnaie acquise par le Musée de Chartres $^{22}$. Ce type XVIII, comme le type IX (Dy 436 ${ }^{23}$ ) n'apparaissent pas dans les trésors et sont donc difficiles à situer précisément. Le trésor de Bonn, bien qu'enfoui au début du XIV ${ }^{\mathrm{e}} \mathrm{s}$. avec un sous-ensemble de monnaies de billon clos dans les années 1290, ne contient qu'une monnaie ancienne, légère et usée de Chartres du type VI et une pièce du type VIII (Dy 434), déjà attesté dans le trésor de Gisors (Eure), dans les années 1240, mais qui est également représenté dans le trésor de Mansigné constitué vers 1306. Celui-ci présente les trois derniers types de deniers anonymes : le type VIII (Dy 434) avec trois pieux entre deux besants, le type XI au lis entre deux besants, représenté dans les trésors des années 1290 comme Annebecq (Eure) et Saint-Léger, mais qui figure encore à Bois-le-Duc vers 1305 (Rappelons qu'entre son achat à Jeanne de Châtillon en juillet 1286 et sa concession à Charles de Valois le comté de Chartres est entre les mains du roi.) et le type $\mathrm{X}$ à la croisette entre deux besants qui est attesté dans des trésors un peu plus tardifs comme Saint-Valery (v. 1310) ou Charost (Cher, après 1296) où apparaissent déjà des pièces au nom de Charles (1293-1328). Celles-ci permettent de dater les trésors de Bressuire (DeuxSèvres) sinon celui de Beauvais-sur-Matha (Charente-Maritime) qui contient des doubles des années 1296-1305. Dans le trésor de Charost la monnaie de Charles est du même type XIV-XV que celles qui figurent aussi dans le trésor de Mansigné : type XIV (Dy 445-446). À Saint-Valery les pièces de Charles correspondent d'une part au

21. F. Dumas, Le monnayage de ducs de Bourgogne, Wetteren, 1983.

22. De même sont récemment passés en vente des types inédits du monnayage angevin de ce prince et il convient de se livrer à un examen très minutieux des émissions de cette période.

23. Les références Dy renvoient à J. Duplessy, Les monnaies françaises féodales, I, Paris, 2004. 
type XII (Dy 442) avec une rosette et deux besants et un T annelé et d'autre part au type XVI marquant le retour aux trois besants (Dy 440). Les trésors plus tardifs de Saint-Maixent (Vienne, vers 1315-1322) et de Manderen-Sierck (Moselle, vers 1328) associent eux aussi des pièces de ces types XIV, XII et XVI.

La séquence que suggère l'enchaînement des trésors serait donc la suivante : type VIII (vers 1240), type XI (peu avant 1290), type X (vers 1295) avant que Charles ne commence (vers 1300) à monnayer à son nom, aux types XIV-XV avec lis en cantonnement de la croix du revers puis au type XII et au type XVI, sans lis. Le lis reparait en cantonnement de la croix sur la variante du type XVI illustrée par le règlement de 1315. Celle-ci constituerait ainsi un type supplémentaire si des exemplaires en étaient retrouvés. Elle présente de plus une légende longue CARTIS CIVIS qui n’apparaît que sur les piéforts agrandis (types XII, XVI et XVII).

Les effectifs et les états de conservation des différents types réunis dans le trésor de Mansigné s'accordent assez bien avec cette proposition de classement qui, de plus, associe tous les piéforts connus aux émissions les plus tardives, postérieures à 1305. Le monnayage s'interrompt au plus tard le 14 mai 1319 lors de la vente au roi par Charles de Valois de son droit monétaire à Angers et à Chartres.

\section{Châteaudun}

Pour le monnayage de Châteaudun, J. Duplessy avait proposé un classement fondé sur la succession des trésors qui est pour l'essentiel repris par S. Michon. Celui-ci distingue deux périodes d'émission pour le type (Dy 468-469) que J. Duplessy situait au tournant des $\mathrm{X}^{\mathrm{e}}-\mathrm{XI}^{\mathrm{e}} \mathrm{s}$. et dont la reprise est possible au milieu du XII ${ }^{\mathrm{e}}$ siècle (types $\mathrm{X}$ et XV). On relève surtout le déplacement à la fin du XIII ${ }^{\mathrm{e}} \mathrm{s}$. des types XXXII-XXXIII que J. Duplessy (Dy 488-489) plaçait avant les émissions aux noms des vicomtes. Ce classement rompt avec le modèle suivi jusqu'à J. Duplessy qui postulait que, comme à Chartres, toutes les émissions anonymes étaient antérieures aux émissions portant le nom des vicomtes. Ce nouveau classement est tout à fait conforté par la présence de cinq pièces du type voisin XXXV-XXXVI (Dy 486) dans le trésor de Mansigné. En effet, dans les trésors du XIII ${ }^{\mathrm{e}} \mathrm{s}$. les monnaies anonymes sont quasiment absentes entre les trésors des années 1215 (Notre-Dame-d'Oé, Alençon...) jusqu'au trésor mal décrit de Châteauroux ou Charost (postérieur à 1296) et aux trésors du début du XIV ${ }^{\mathrm{e}}$ s. pour lesquels ces types anonymes ne sont souvent pas décrits : trésors de Rocamadour (Lot, vers 1306), de Saint-Marcel-de-Félines (Loire, après 1311) et de Saint-Jean de Brévelay (Morbihan, vers 1314-1317)..., tous postérieurs à celui de Mansigné. Le trésor de Saint-Léger-des-Bois (Maine-et-Loire, vers 1290-1295) ne contient encore que des pièces du vicomte Raoul (1264-1302) de même que celui d'Aurimont (Gers, vers 1305), contemporain de Mansigné, mais sensiblement plus éloigné de l'atelier de Châteaudun. Le trésor de Bonn (vers 1290) ne contient, une fois encore, qu'une monnaie assez ancienne, au nom des vicomtes Geoffroy (1215-1253) et ne contribue guère au classement de ces monnayages. Dans les trésors du début $\mathrm{du} \mathrm{XIV}^{\mathrm{e}} \mathrm{s}$. où les monnaies anonymes sont décrites, elles correspondent effectivement aux types XXXII-XXXIX de S. Michon : les trésors de Saint-Valery (vers 1310) et de Saint-Maixent (Vienne, vers 1315-1322) comptent des exemplaires au type XXXIV (Dy 488) de même que celui de Manderen Sierck (vers 1328) qui y associe 
des pièces avec tête à gauche du type XXXIX (Caron, Pl. 5, 21), soit précisément le type illustré dans le règlement de 1315. Le trésor de Manderen contient également une monnaie du vicomte Guillaume (1302-1315) similaire à celle du trésor de Mansigné (type XXXVII). Celui-ci dont le terminus se situe vers 1305-1306 vient assurer que cette émission prend place au début du monnayage de Guillaume, entre 1302 et 1305. Faut-il pour autant supposer que toutes les émissions anonymes sont postérieures à cette émission? Cela conduirait à décaler la date d'enfouissement du trésor de Mansigné vers 1305, le temps que soit également frappé le type anonyme XXXVXXXVI qui y est aussi représenté, même si celui-ci semble correspondre, du fait de sa rareté, à une brève émission. On peut s'interroger sur la position de l'émission au nom de Guillaume puisque l'exemplaire semble plus usé que les deniers anonymes dans le trésor de Mansigné. Ce retour aux émissions anonymes vers 1305 pourrait s'inspirer (avec deux croissants et un besant) des derniers types anonymes frappés en abondance (types XVII-XVIII, Dy 483-485) qui ont circulé en grandes quantités aux temps du conflit entre Philippe Auguste et les Plantagenêts. Cette rupture typologique pourrait également être associée au retour à la bonne monnaie engagé par le roi à partir de 1305. Le meuble qui cantonne la croix est peut-être à rapprocher de celui qui figure sur les monnaies bretonnes de Guingamp à l'époque de Jean II (1286-1305), mais il apparaît aussi sur des pièces de Bourbon vers $1210 \ldots$, ce qui n'apporterait pas d'indice chronologique très précis.

Les trésors montrent qu'après les émissions de types variés signées par Geoffroy (après 1215), Robert (1253-1264) types XXVI-XXIX, Simon, type XXX, Raoul (1264-1302) type XXXI, et peut-être Guillaume (type XXVII entre 1302 et 1305) reprennent, avant 1305 des émissions anonymes au type de la tête : types XXXVXXXVI puis XXXIV et XXXIX, pour lesquelles sont connus des piéforts: type XXXIV et XXXVIII de module agrandi.

\section{Vendôme}

Les émissions anonymes qui s'enchaînent depuis le $\mathrm{x}^{\mathrm{e}} \mathrm{s}$. circulent jusque dans les années 1210 avec le type XIII (Dy 545) à légende déformée VDONCAOSTO qui figure encore dans les trésors d'Alençon (Orne), de Vallon-sur-Gée (Sarthe) ou de Veniers et Gençay (Vienne). Ensuite sont attestées des émissions du type XIV (Dy 553) présentant une légende Vindocino et le nom d'un comte Jean, moins probablement Jean II (1202-1211) ou même Jean III (1211-1217) que Jean IV (1217-1240), inspirées par le type tournois. Le passage à des émissions signées par les comtes et l'abandon du type à la tête semble contemporain à Châteaudun et à Vendôme. Ces pièces du type XIV apparaissent dès les trésors de Senillé (Vienne) vers 1223 et de Kessab en Orient (vers 1225-1230). Le type XVII (Dy 556) également au nom de Jean, mais à légende Vidocinensis, figure dans le trésor de Ladignac (Haute-Vienne) avec des monnaies similaires de Pierre (1240-1249) du type XVIII (Dy 561) qui datent le trésor. Le trésor de Bouvron (Loire-Atlantique, vers 1241-1245) contenait également un denier de Jean au type XVII (Dy 555) mais aussi une pièce d'un type anonyme type XXII (Dy 560) qu'il est de ce fait difficile de situer vers 1266 comme le fait S. Michon en écartant délibérément le témoignage des trésors. Il faut probablement en rapprocher les pièces de même revers portant le nom de Jean du type XXVIII 
(Dy 559) qui ne sont attestées dans aucun trésor. Ces deux émissions de date peu discutable viennent assurément rompre la continuité des types XVII, XVIII, XXI aux noms de Jean, puis de Pierre, puis de Bouchard VI (1240-1271) en introduisant, de plus, une émission anonyme dans la séquence de ces frappes qui sont toutes à légende Vidocinensis. En effet, dans les trésors de la seconde moitié du XIII ${ }^{\mathrm{e}} \mathrm{s}$. comme Jonzac (Charente-Maritime), Caudebec (Seine-Maritime) et Beaugency (Loiret) s'enchaînent et se succèdent les émissions de Jean (type XVI-XVII), de Pierre (type XVIII Dy 561), de Bouchard VI (type XXI Dy 565). À ce type XXI de Bouchard le trésor de Vernou (Indre-et-Loire, vers 1280-1290) associe des pièces de Jean à légende Vidocinensis (type XXV Dy 567) attribuables à Jean V (1271-1313) mais aussi d'autres pièces de Jean à légende Vedome castr (type XXIV Dy 552). Les deux types XXV et XXIV figurent dans le trésor de Mansigné (vers 1305) mais le type XXIV est seul présent dans celui de Charost (Cher après 1296) comme dans le trésor de Bonn (Dy 561 croisette en 2) où l'exemplaire est décrit comme en bon état. Pour le monnayage de Vendôme le trésor de Bonn apporterait un jalon intéressant : cette pièce serait proche du terminus vers 1296 du sous-ensemble des monnaies de billon du trésor qui est donné par la présence d'un double tournois de Philipe IV alors que la plupart des autres monnaies bléso-chartraines qu'il contient appartiennent à des émissions du milieu $\mathrm{du} \mathrm{XIII}^{\mathrm{e}} \mathrm{s}$. Comme ce sont deux types à légende Vedome castr (types XXIV et XXXI) qui sont ensuite associés à Saint-Valery (vers 1310), il semble possible d'intervertir l'ordre de succession des types proposé par S. Michon : type XXV (Dy 567) puis type XXIV (Dy 552). Le type XXXI (Dy 549) à légende Vedome castr attesté à Saint-Valery marque la reprise des émissions anonymes au type de la tête qui sont peut-être déjà présentes dans les trésors mal décrits de Rocamadour (Lot vers 1306), de SaintMarcel-de-Félines (Loire, vers 1311) ou de Saint-Jean-de-Brévelay (Morbihan vers 1314-1317). La reprise des émissions anonymes et du type de la tête apparait peutêtre un peu plus tardive qu'à Châteaudun, puisque le trésor de Mansigné n'en contient aucun exemplaire. Ce type XXXI assurément antérieur à 1311 correspond au dessin du règlement de 1315, si ce n'est que ce dessin présente un besant en cantonnement de la croix du revers comme l'obole qui constitue le type XXVII de S. Michon et qui, pour le reste, correspond à l'obole du type XXXI (Dy 550) ${ }^{24}$. Le trésor de Manderen Sierck enfin, contient à côté des pièces anciennes au type XVI (Dy 555 et 557) de Jean IV (1218-1239) qui figurent aussi dans celui de Bois-le-Duc et des types XXV (Dy 567) et XXIV (Dy 551) de Jean V (1271-1315), des deniers anonymes (type XXXI Dy 549) et enfin un denier de Bouchard avec une légende Bo Comes / Vidocinensis et un type au chatel accosté de lis (type XXX, Dy 569) qui pourrait succéder à une émission similaire de Jean (1313-1315) type XXIX (Dy 568) qui n'apparaît dans aucun trésor. L'apparition de ce type seulement dans un trésor tardif suggère une attribution à Bouchard VII (1315-1369) et une reprise d'émissions signées et de la légende Vidocinensis. Le règlement de 1315 aurait alors prévu la reprise du type anonyme frappé quelques années plus tôt, vers 1310. Toutefois l'exemplaire du trésor de Manderen semble usé et une attribution de cette pièce à Bouchard VI (1249-1270) rétablirait davantage de continuité dans les légendes (avec la forme Vidocinensis) et 
son type au chatel accosté de lis serait à rapprocher du type XXII présent dans le trésor de Bouvron (vers 1241) et du type XXVIII, le type XXIX étant alors à attribuer à Jean IV (avant 1240). Cette hypothèse aurait de plus l'avantage de réduire les alternances entre types anonymes et types signés, entre légendes Vidocinensis et Vedome Castr et entre types à la tête et types au chatel qui donne au monnayage de Vendôme une extrême complexité si on s'en tient au classement proposé par S. Michon. Il est vrai que rien ne garantit que la logique ait présidé à l'organisation et à la succession des émissions à une époque où s'enchaînaient les affaiblissements de Philippe le Bel. Le seul piéfort authentique mentionné serait au type de l'avis de 1315 qui marquerait la fin du monnayage de Vendôme, la fabrication s'interrompant avant même la vente par Bouchard de son droit de monnayage en 1320.

Il faut observer en tout cas que les trésors ne permettent pas de situer quelques émissions rares (types XXVI-XXVII, proches du type XXXI) et le type XXIII à légende Vindocino.

Blois

Les monnaies portant les noms des comtes commencent avec Jean (1241-1279) dont les deniers au type du chatel (type X Dy 586) apparaissent dans les trésors de Saint-Clair-sur-Elle (après 1253) et de Bretagne (après 1266), alors que le trésor d'Argenton-sur-Creuse (Indre, après 1271) contient encore un denier anonyme du type IX (Dy 585, avec une croisette entre deux besants). Les monnaies au nom de Jeanne (1279-1292) apparaissent dans les trésors des années 1280 comme celui de Vernou (vers 1280-1290) ou d'Angers (vers 1285). Toutes ces pièces à légende $I$ comitissa appartiennent au type XII (Dy 587). Dans les années 1290 on trouve toujours des monnaies de Jeanne : une obole à Bressuire (Deux-Sèvres, après 1293) et des exemplaires à Châteauroux ou Charost (après 1296) qui sont associés à des monnaies au nom d'Hugues (1292-1303) correspondant aux types XIII, XIV et XVI (Dy 589 à 592). À Beauvais-sur-Matha (après 1296) il y a seulement une obole d'Hugues (type XIII), mais à Saint-Léger (vers 1290) reparaissent des pièces anonymes, avec sept exemplaires du type XI (Dy 584, avec un lis entre deux besants) et dans le trésor de Saint-Maurice-le-Girard (Vendée, début XIV ${ }^{\mathrm{e}} \mathrm{s}$.) figurent également des monnaies anonymes, non décrites. Le trésor de Bonn (vers 1296) contenait également une obole anonyme de ce type XI (Dy 585) associée à cinq deniers anciens très usés du type VIII (Dy 576-580) et à un denier de Jeanne du type XII (Dy 587) en bon état.

Le trésor de Mansigné propose en quelque sorte une récapitulation de ces différentes émissions avec des pièces de Jean au type $\mathrm{X}$ (un exemplaire usé), de Jeanne au type XII (trente exemplaires) et de Hugues (cinq exemplaires du type XVI et seize exemplaires, très frais, du type XIII qui pourrait être plus récent) mais aussi des émissions anonymes des types VIII (un exemplaire usé), IX (quatre exemplaires), XI (huit exemplaires) et XV (un exemplaire usé). Comme les émissions de Gui de Châtillon (1303-1342) ne sont pas encore représentées à Mansigné dans l'abondante série des monnaies blésoises du trésor - ce qui contribue à fixer le terminus du trésor assez près de 1303, vers 1305 - il faut conclure que les divers types anonymes ont précédé (types VIII, IX?) ou se sont intercalés entre des émissions des comtes. C'est probablement le cas en particulier pour le type XI, avec un lis entre deux besants qui est 
également attesté dans le trésor de Saint-Valery (vers 1310) à côté de monnaies de Gui du type XVII (Dy 593-594). Ce type, qui apparaît vers 1290 s'inscrit assez bien en parallèle des émissions chartraines au même type, mais il faut rappeler qu'à Blois le lis figure déjà sur les monnaies de Jeanne (1271-1292).

Pour le type XV, l'état de conservation de l'exemplaire du trésor de Mansigné ne correspond pas à ce que l'on attendrait pour une pièce marquant l'émission terminale du trésor, en 1303, si on suit le classement de S. Michon. Ce fait renvoie plutôt au classement antérieur qui le rapprochait du type XI au lis anonyme dont il se différencie seulement au revers, par le cantonnement de la croix. Le trésor de Beaugency daté vers 1270 qui est le seul à présenter des deniers et oboles de ce type en devient moins déroutant.

En revanche ce trésor inciterait à intervertir le classement des deux principales émissions de Hugues : le type XVI qui reprend et poursuit le type XII de Jeanne autant qu'il est repris par le type XVII de Gui, venant avant le type XIII. Ce type XIII de Hugues (1292-1303) pourrait constituer le plus récent des deniers blésois de Mansigné.

Des monnaies de Gui (1303-1328) non décrites figuraient déjà dans le trésor de Rocamadour (Lot, après 1306) et à Saint-Marcel de Félines (Loire, après 1311), à Vannes (vers 1315-1317) les descriptions, imparfaites, ne permettent pas de distinguer entre les types XVII-XIX, ou, à Villeneuve (Puy-de-Dôme, vers 1315-1322) entre les types XVII ou XVIII (Dy 593-595). Pour le trésor de Saint-Maixent (vers 1315-1322) les renvois bibliographiques à l'ouvrage ancien de P. A. Tobiesen Duby ${ }^{25}$ permettent de reconnaître les types XVII (Dy 593) et XVIII (Dy 595) de Gui associés à des monnaies de Hugues du type XIII (Dy 590) et à des monnaies anonymes : quatorze exemplaires de cinq variétés alors que l'ouvrage de Duby n'en illustre que trois, correspondant aux types VIII et IX. À Saint-Jean de Brévelay (vers 1315-1317) on compte encore sept deniers de Jean et deux oboles de Jeanne pour dix-sept deniers de Gui répartis en deux types et trois variétés. Dans le trésor de Manderen Sierck (vers 1328) les seules monnaies anonymes sont anciennes (cinq deniers de type VIII, Dy 579) et accompagnées de monnaies de Jean (cinq deniers de type X Dy 586), de Jeanne (un denier de type XII, Dy 587) avec seulement un denier et huit oboles de Gui (type XVII, Dy 593-594). C'est encore le trésor de Saint-Valery qui apporte la meilleure information sur le classement des émissions de Gui en attestant que le type XVII a été frappé avant 1310. La période finale des émissions blésoises avec des émissions décrites par les comptes en 1316 et jusqu'à la vente de la monnaie en mai 1328 ne sont pas bien connues, mais c'est bien là que doit être placé le type XIX avec un B en cantonnement de la croix qui n'apparaît pas de façon certaine dans les trésors mais qui est illustré dans le règlement de 1315. Des piéforts de denier et d'obole correspondent aux émissions précédentes de Gui (type XVIII pour le denier, XVII pour l'obole) attestées dans des trésors postérieurs à 1315 .

Toutefois on connaît un piéfort du type anonyme IX avec une croisette entre deux besants émis avant les années 1270, selon le témoignage du trésor d'Argenton-surCreuse, ce qui le rapproche chronologiquement des plus anciens piéforts royaux 
de Louis IX et Philippe III. L'abondance dans les trésors tardifs, et seulement dans ceux-ci, de ces types anonymes qui présentent une tête chargée de trois besants, selon un modèle chartrain, sous laquelle figurent, entre deux besants, trois barres (obole du type VIII), un lis (type XI) ou une croisette (type IX) reste cependant surprenante. Au vu des trésors les émissions parallèles de Chartres sont respectivement datables des années 1240 (barres), d'avant 1290 (lis) et d'avant 1295, pour le type à la croisette. Le parallélisme des émissions qui se situent au moment où, à partir de 1286 les comtés relèvent de princes différents ne se vérifie donc pas très clairement. Mais surtout, le monnayage de Blois illustre au moins dans les années 1270-1290 un entrecroisement d'émissions anonymes et d'émissions signées qui résiste aux tentatives de rationalisations des émissions qui ont été tentées ci-dessus pour les productions de Chartres, de Châteaudun et de Vendôme. L'enquête reste donc ouverte. 